\title{
Planning and Analysis of Fiber Wrap Trajectory in Pressure Vessel
}

\author{
Wenzhi Liü, a Mingjun Huang², Jiancheng Tian² and Ye Chen² \\ ${ }^{1}$ College of Aviation, Inner Mongolia University of Technology Huhhot 010051, China. \\ ${ }^{2}$ College of Mechanical Engineering, Inner Mongolia University of Technology Huhhot 010051, \\ China.
}

a1733924013@qq.com

\begin{abstract}
Keywords: Robot, Composite pressure vessel, Geodesic winding line type, Winding trajectory planning.
\end{abstract}

\begin{abstract}
According to the application requirements of a kind of composite pressure vessel, aiming at the fiber winding of multi-axis CNC filament winding machine based on robot platform, the cylinder body and head of pressure vessel are analyzed. The geodesic winding line. Using MATLAB to calculate the trajectory of robot winding equipment under the parameters of constant free fiber length and geodesic winding. The stability of the robot during the winding process is analyzed to provide the basis for the fiber winding experiment.
\end{abstract}

\section{Introduction}

Fiber winding technology is the key technology of composite material pressure vessel winding [1, 2], and is widely used in aerospace, petroleum, chemical and other industries. Fiber wound pressure vessel with high strength, structural stability, can effectively reduce the quality of the container [3], $\mathrm{CNC}$ fiber winding machine is the pressure vessel filament winding molding equipment. With the rapid development of industry, the domestic six-axis fiber winding machine cannot continuously improve the performance of composite pressure vessels, which puts forward higher requirements on the technical performance of the process and equipment of the fiber-wound composite pressure vessel [4]. In order to meet the increasingly high requirements of fiber wound pressure vessel performance, in view of the current level of domestic technological development and the international environment, research and development based on the robot platform dynamic CNC fiber winding machine composite material pressure vessel fiber winding is an urgent need to be resolved.

In this paper, the winding pattern of fiber-wound composite material pressure vessel based on robotic platform was designed under the condition that the design of complete machine was completed. The geodesic winding model of the pressure vessel was analyzed. The doffing point and winding center angle of the relationship, through the MATLAB in the constant free fiber length, geodesic winding and other parameters of the robot winding device trajectory calculation. Analysis of robot stability during the winding process.

\section{Composite Pressure Vessel Wound Wire Design}

The forming process and performance of fiber-wound composite pressure vessels are determined by the winding pattern [5]. For the pressure vessel fiber winding based on the robot platform, the liner design of the cylinder body and the elliptical head composite rotor core is performed.

\subsection{Pressure Vessel Barrel Measuring Ground Wire Winding}

The geomorphological surface of the cylinder winding track is a spiral, the mathematical model shown in Figure1, set point $\mathrm{Q}$ is the starting point of winding doff, $\mathrm{M}$ points for the winding process in the doffing of the fiber surface in the cylinder Point, the projection of the point $\mathrm{M}$ on the bottom surface is the point $\mathrm{M} 1, \mathrm{R}$ is the radius of the cylinder, a cylindrical coordinate system as shown in the figure is established, the point $\mathrm{Q}$ is on the $\mathrm{x}$-axis, the angle $\theta 1$ is the starting doffing point $\mathrm{Q}$ in the winding process The point $\mathrm{M}$ of the mandrel core angle, $\mathrm{h}$ is the fiber from the $\mathrm{Q}$ point at a winding angle $\varphi 1360^{\circ}$ after the distance between the point $\mathrm{Q}$ and $\mathrm{Q}, \mathrm{L}$ is the length of the cylinder, there is a container body surface The geodesic equation is 


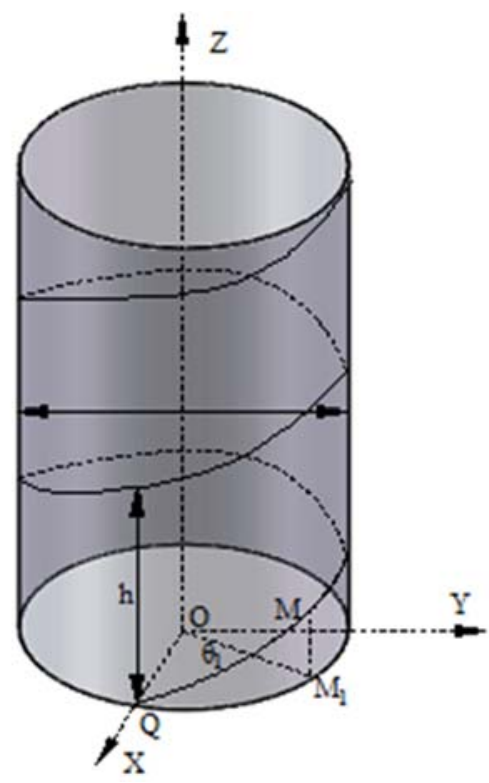

Fig. 1 The geodesic on the surface of the cylinder

Cylinder core mold angle is:

$$
\left\{\begin{array}{l}
x=R \cos \left(\theta_{1}\right) \\
y=R \sin \left(\theta_{1}\right) \\
z=\left(\theta_{1} R\right) \cdot \tan \left(\varphi_{1}\right)
\end{array}\right.
$$

$$
\theta_{t}=\frac{L \cdot \tan \left(\varphi_{1}\right)}{2 \pi R} \times 360^{\circ}
$$

\subsection{Relationship Between Center of Winding Angle and Doffing Point of Geodesic Oval Head}

As shown in Fig2, the locus of the doffing point on the surface of the pressure vessel head is a geodetic line[6,7].

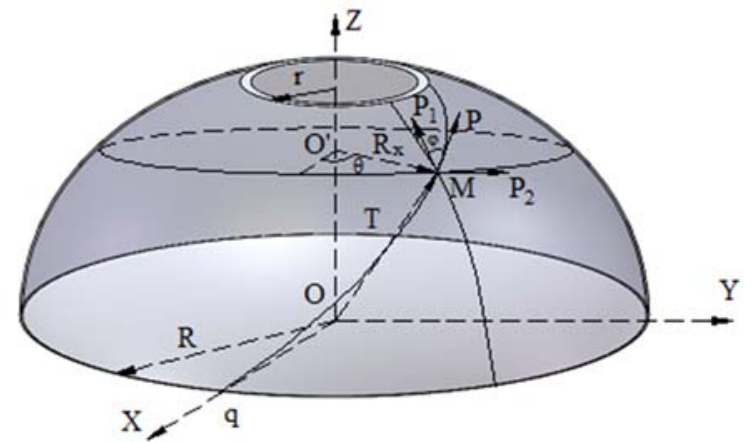

Fig. 2 Oval head winding model

The geodesic equation of the surface of the elliptical head is as follows:

$$
\left\{\begin{array}{l}
X=R_{x} \cos (\theta) \\
Y=R_{x} \sin (\theta) \\
Z=b \sqrt{R^{2}-R_{x}^{2}} / R
\end{array}\right.
$$

The radius of the doffing point at any point on the elliptical head and the winding center angle $\theta$ satisfy the following relation:

$$
\theta=\int_{R_{x}}^{R} \frac{r \cdot \sqrt{R^{4}+R_{x} \cdot\left(b^{2}-R^{2}\right)}}{R \cdot R_{x} \cdot \sqrt{R^{2}-R_{x}^{2}} \cdot \sqrt{R_{x}^{2}-r^{2}}} d R_{x}
$$

In formulas (3) and (4), Rxis the radius passing through the doffing point $M$ and parallel to the circle at the bottom of the seal, and $b$ is the semi-major axis of the ellipse and $r$ is the radius of the seal. 


\subsection{Coordinate Analysis of Mandrel Relative to Nozzle}

In the robot winding process equipment, the nozzle is stationary, the robot drives the winding machine to move. As shown in Figure 3 o point for the nozzle position to o point as the origin of the establishment of the coordinate system o (x,y, z) to $\mathrm{O}$ point to establish the coordinate system $\mathrm{O}$ (X, $\mathrm{Y}, \mathrm{Z})$, the head The center coordinates of the bottom of the $\mathrm{O}(\mathrm{x} 0, \mathrm{y} 0, \mathrm{z} 0), \mathrm{y} 0$ is a constant.

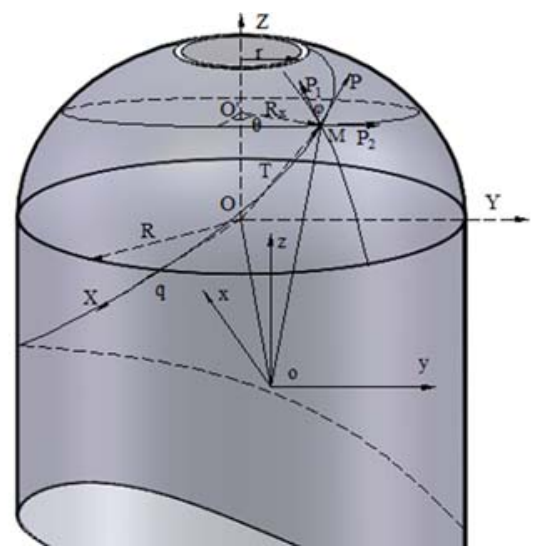

Fig. 3 The core mode is relative to the silk mouth coordinates

It can be seen from Fig 3 that the mandrel coordinates in the process of robot winding motion satisfy the following relation:

$$
\begin{gathered}
x_{0}=R_{x} \cos (\theta)+\frac{\lambda R \cos (\theta) \cos (\varphi) \sqrt{R^{2}-R_{x}^{2}}}{\sqrt{\left(b R_{x}\right)^{2}+R^{2}\left(R^{2}-R_{x}^{2}\right)}}+\lambda \sin (\theta) \sin (\varphi) . \\
z_{0}=-\frac{b \sqrt{R^{2}-R_{x}^{2}}}{R}+\frac{b \lambda R_{x} \cos (\varphi)}{\sqrt{\left(b R_{x}\right)^{2}+R^{2}\left(R^{2}-R_{x}^{2}\right)}}
\end{gathered}
$$

In Eqs. (5) and (6), $\lambda$ is the free fiber length.

\section{Robot Winding Trajectory Planning}

The relationship between the doffing point and the center angle of the winding is obtained. The winding of the robot with a constant free-fiber length is calculated for the winding of the robotic composite pressure vessel.

\subsection{Analysis of the Motion Trajectory of Winding Equipment}

A complete winding cycle of a pressure vessel can be divided into six stages. During the winding process, each stage of the robot's end effector has corresponding trajectories in the range of motion space. According to the theoretical calculation, when the length of the free fiber is constant, the trajectory of a robot has a unique solution.

\subsection{Parameterized Modeling of Robot D-H}

The basis of D-H parameterized modeling is to establish a connecting rod coordinate system at the joints of each connecting rodto obtain 4 parameters are obtained to determine the geometric characteristics of the motion of the robot rod: the joint rotation angle $\theta$ ifrom the $\mathrm{Xi}-1$ axis to the $\mathrm{Xi}$ axis around the $\mathrm{Zi}-1$ axis (according to the right-hand rule); the angle $\theta \mathrm{i}$ from the $\mathrm{Xi}-1$ axis to the $\mathrm{Xi}$ axis along the $\mathrm{Zi}-1$ axis Offset distance di; the distance from the $\mathrm{Zi}-1$ axis to the $\mathrm{Zi}$ axis along the $\mathrm{Xi}$ axis is the length ai of the rod; the $\mathrm{Zi}$ axis is the torsion angle aiof the rod about the Xiaxis (by righthand rule) from the $\mathrm{Zi}-1$ axis to the $\mathrm{Zi}$ axis. The $\mathrm{D}-\mathrm{H}$ modeling principle, the establishment of robot link diagram, shown in Figure 4. 


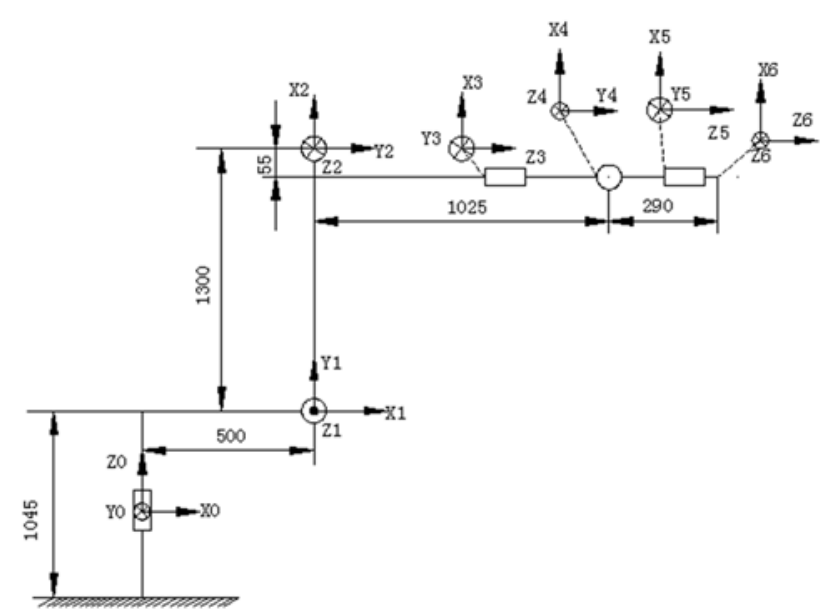

Fig. 4 Connecting rod sketches of a robot

From the four parameters of the robot link, the coordinate system at each joint is introduced into the homogeneous transformation matrix[8]:

$$
A_{i}=\left[\begin{array}{cccc}
\cos \theta_{i} & -\sin \theta_{i} \cos \alpha_{i} & \sin \theta_{i} \sin \alpha_{i} & a_{i} \cos \theta_{i} \\
\sin \theta_{i} & \cos \theta_{i} \cos \alpha_{i} & -\cos \theta_{i} \sin \alpha_{i} & a_{i} \sin \theta_{i} \\
0 & \sin \alpha_{i} & \cos \alpha_{i} & d_{i} \\
0 & 0 & 0 & 1
\end{array}\right]
$$
1.

From Figure 4, we can get the geometrical parameters of each rod of the robot, as shown in Table

Table 1D-H parameters of a robot

\begin{tabular}{ccccc}
\hline Joint $i$ & $d_{i}(\mathrm{~mm})$ & $a_{i}(\mathrm{~mm})$ & $\alpha_{i}\left({ }^{\circ}\right)$ & $\theta_{i}\left({ }^{\circ}\right)$ \\
\hline 1 & 1045 & 500 & $90^{\circ}$ & 0 \\
2 & 0 & 1300 & $180^{\circ}$ & $90^{\circ}$ \\
3 & 0 & 55 & $-90^{\circ}$ & 0 \\
4 & 1025 & 0 & $90^{\circ}$ & 0 \\
5 & 0 & 0 & $90^{\circ}$ & $180^{\circ}$ \\
6 & 290 & 0 & 0 & 0 \\
\hline
\end{tabular}

The robot's initial position is $(0, \mathrm{pi} / 2, \mathrm{pi}, 0, \mathrm{pi}, 0)$ using the connecting rod parameters in Table 1 . In the MATLAB environment, the robotics Toolbox toolbox is used to draw the three-dimensional structural model Show.

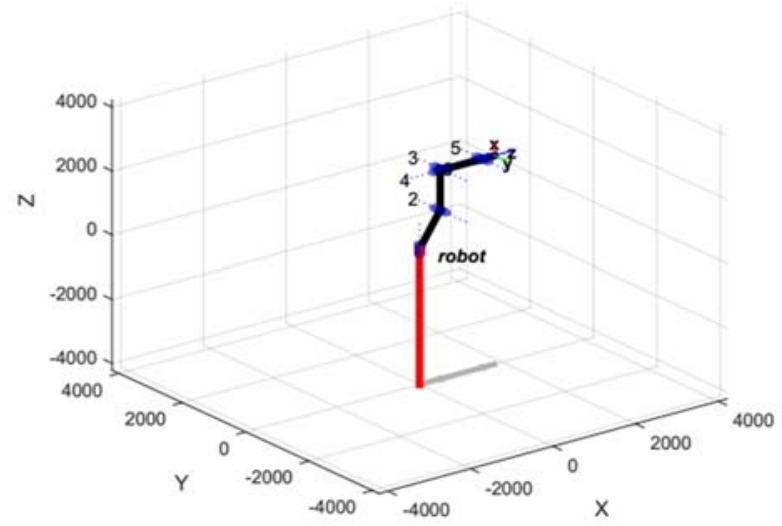

Fig 5. 3D digital structure model of robot

\subsection{Robot Trajectory Planning}

In the condition that the length of free fiber is constant, Using the geodesic winding method, we use circular interpolation, linear interpolation and three spline interpolation interpolation algorithm to track a complete cycle winding, and get the trajectory of the robot's terminal. The robotics toolbox is used to find out the kinematic inverse solution to get the first 3 joints in the process of robot winding 
The angular displacement, angular velocity and time curve. The initial winding angle of the robot winding device is $(0,-$ pi $/ 2$, pi, $0,0,0)$.
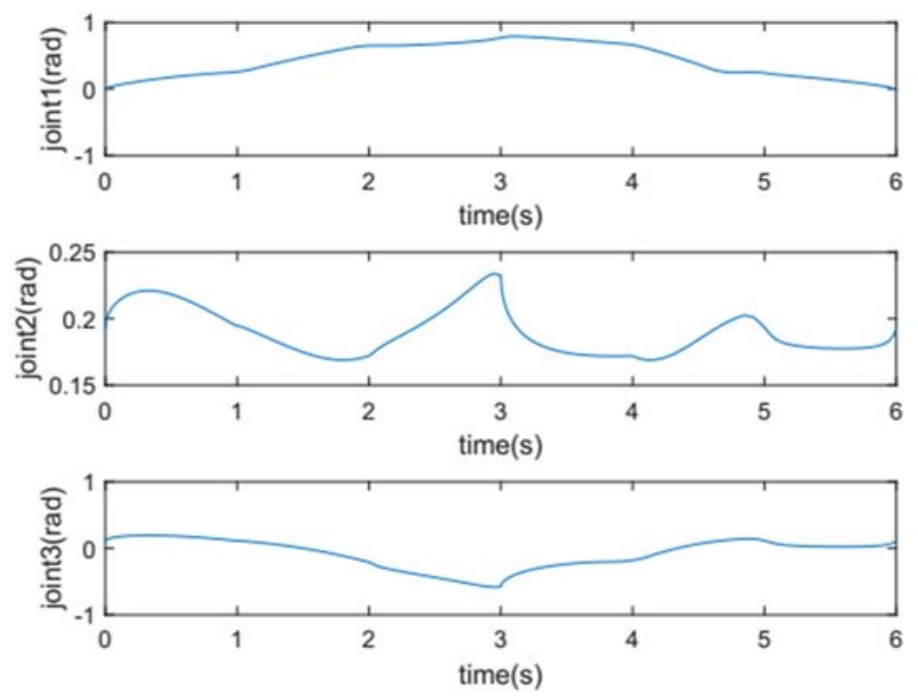

Fig. 6 The relationship between the 3 joint displacementand time in the front of the robot
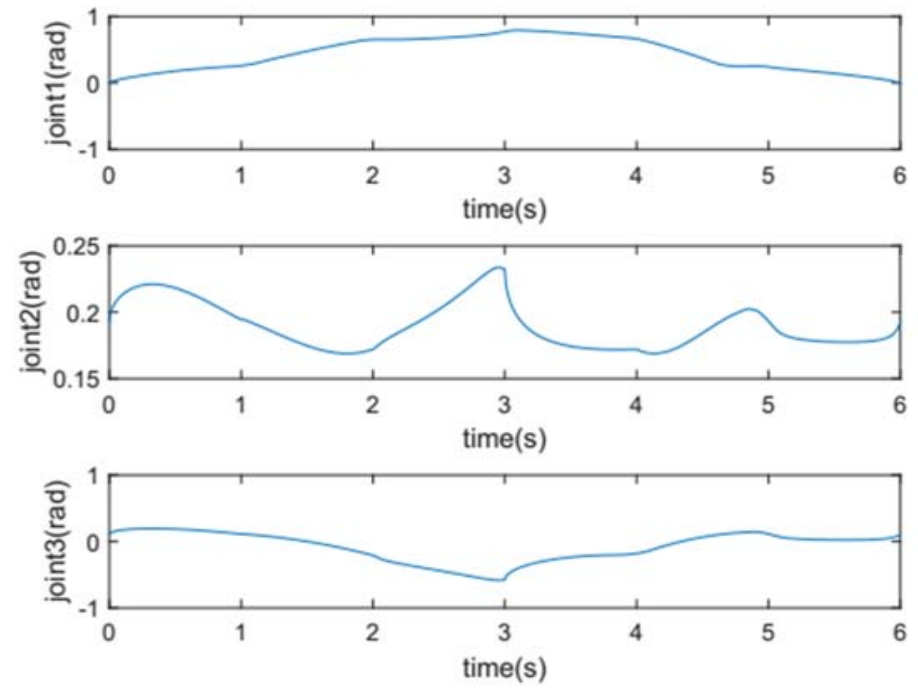

Fig. 7 The relationship between the velocity and time of the 3 joint angles of the front of the robot

It can be seen from Fig 7 that the joint speed changes steadily and the speed does not change suddenly when the robot wraps the container. The winding device will not produce obvious tremor and jitter, so the robot trajectory meets the requirements in a complete cycle.

\section{Conclusion}

Through the analysis of the cylinder of the composite material pressure vessel and the geodesic winding model of the head, the doffing point trajectory is obtained. By calculating the winding trajectory with the constant free fiber length of $200 \mathrm{~mm}$, the robot winding equipment works smoothly, No large vibration, in line with the expected results, the winding trajectory can be applied to the robot winding experiment.

\section{Acknowledgements}

The support of key scientific research project of the Inner Mongolia Autonomous Region University (NJZZ18075) is gratefully acknowledged.

The support of science research project of teachingreform of Inner Mongolia University of Technology (2013001) is gratefully acknowledged. 


\section{References}

[1] Cai Jingang, YU Baifeng, Yang Zhizhong, Pei Fang. The development and status of filament winding technology and industry in China. FRP / composites.(2014) No. 09, p. $42-51$.

[2] Wang Yingqi, Gai Dengyu, song Yiguo. Current situation and development trend of filament winding technology. materials review. Vol. 25 (2011) No. 05, p. 110-113.

[3] Lei Haifeng. Introduction of domestic aramid fiber composite test container and its properties. propulsion technology.(1990) No. 04, p. 40-46.

[4] Xu Jiazhong, Yang Hai, Gao Liangchao, Li Ying, Wang Yan. Optimization of composite material winding strategy based on robot. Journal of composite materials, Vol. 33 (2016) No. 06, p. 13181326.

[5] Leng Xingwu. Design of filament winding line type. Application of engineering plastics. (1983) No. 02, p. 24-31.

[6] AlfredGray,Elsa Abbena, Simon Salamon. Modern Differential Geometry of Curves and Surfaces with Mathematic. CRC Press,1993, p. 156-178.

[7] Xu Jia-zhong, Jia Chunming, Zhang Sibo. Fiber winding method and application based on Parameterized Design. Application of engineering plastics. Vol. 44 (2016) No. 10, p. 52-56.

[8] Chen Gui, Wang Jian-hong, Tang Yu-dong.Research on kinematics simulation and experiment of KUKA robot. Combined machine tool and automatic machining technology. (2014) No. 08, p. 94-97. 